\title{
Analysis of ATE Measurability Using Information Flow Model
}

\author{
Jing Wang ${ }^{1}$, Qi Zhang ${ }^{2}$, Shilin $\mathrm{Wu}^{3}$ \\ Mechatronics and Automation School, National University of Defense Technology, 410073, Changsha, China, \\ rruohanzi2010@163.com, 27hang_Qi_7233@163.com, ${ }^{3}$ gfkdwsl@sina.com
}

\begin{abstract}
Since many test equipments can't be calibrated conveniently, this paper proposed a new concept named measurability to solve the problem. The measurability is defined, and its indexes are given in detail. Selected from the models of testability, the information flow model is used to analyze the ATE (automatic test equipment) measurability. The correlative matrix of information flow model is decided according to the trace chain. Finally, a practical example is given to show the analysis process.
\end{abstract}

Keywords: ATE, measurability analysis, information flow model, correlative matrix

\section{INTRODUCTION}

$\mathrm{T}$ HE CONCEPT of testability refers to the capability of the UUT (unite under test) to be tested. However, we found that the test equipment should also be considered to have the ability to be measured (or tested) conveniently. In order to evaluate the performance of the measure (or test) equipments, we put forward the concept of measurability for the first time, which is driven from the concept of testability. The root word, measure has a broad sense includes metrology, test, calibration and verification for measure (or test) equipments.

We took ATE as the object to explain measurability. When an ATE is used to test a UUT, its capability to test should be measured firstly, which means "the testability of test equipment”. So ATE measurability, which is a comprehensive concept, is its ability to be measured.

Measurability is a design characteristic that evaluates the capability to be measured (or tested) and the guarantee of accuracy for measure (or test) equipments. Measurability design is the process to improve measurability. We proposed the connotation of ATE measurability as the follows:

1) The ATE measurability contains the parameters of design characteristics, such as the measure range and stability, which refers to the ability to measure (or test) a measurand.

2) It reflects the capability to be detected, especially the ability to be diagnosed, isolated, fault location, so the measurability has some indexes of testability.

3) It refers to the convenience to measure and calibrate the test items and parameters, which has the preconditions of calibration accessibility and feasibility. Besides, the calibration interval is another factor to affect the convenience of calibration.

4) Compare with UUT, ATE is the test equipment which can be seen as a standard of measurement. So the accuracy of ATE should be assured.

In order to analyze the measurability, we put forward some indexes include qualitative and quantitative ones as follows (shown in Fig.1):

The qualitative indexes include:

Measure accessibility: reflects the level of difficulty to approach the unit under measure. An equipment or system with good measure accessibility requires little assembling moving and can be done in common environment.
Measure feasibility: can be interpreted as the verification of whether the measurement can be done or not. The measurement plan should be capable of being applied and executed.

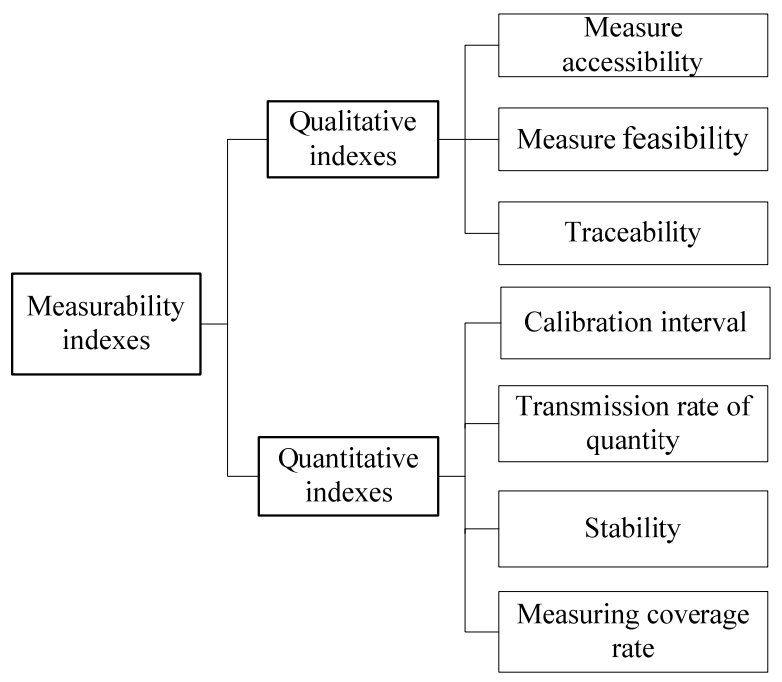

Fig.1. The Measurability indexes

Traceability: refers to an unbroken chain of comparisons relating an instrument's measurements to a known standard.

The quantitative indexes include:

Calibration interval: the time between two consecutive calibrations made on a measure (or test) instrument (or equipment).

Transmission rate of the value of quantity is defined as:

$$
\frac{\text { number of parameters being traced }}{\text { total number of the parameters }}
$$

Stability: the capability of a measuring equipment to keep and maintain the metrology characters as time goes on.

Measuring coverage rate is defined as:

$$
\frac{\text { number of instruments being measured }}{\text { total number of instruments }}
$$

This paper is organized in 4 sections. Section 2 introduces the information flow model of testability. Section 3 presents the reasons why we choose this model for measurability of ATE. A practical example is given in part $B$ of section 3 . The values of measurability indexes are shown in section 4 . 


\section{INTRODUCTION OF INFORMATION FLOW MODEL IN TESTABILITY ANALYSIS}

\section{A. Existing models for testability analysis}

At present, researches toward testability include system modeling, optimization of test and diagnose order, construction of fault-tree, evaluation and analysis of system testability, improvement of system testability, etc. This paper presents an approach to solve the problems about measurability with the same idea of system modeling for testability. The exiting methods of testability modeling are given as follows:

Logic model is composed of function flow graph and dependency chart [1]. The function flow graph, which is denoted by directional chart, describes the transmission paths. On the basis of dependency knowledge, logic model describes the dependency and causality relations to analyze the testability and diagnosis. Information flow model cares about the fault patterns and test items [2]. The dependency relations between faults and tests can be interpreted and correlative matrix can be used to calculate the indexes of testability. Multi-signal flow graphs model connects the faults and tests by function signals instead of relations, which is widely used in testability design and fault diagnosis of system [3-5]. Hybrid diagnostic model, which has the same idea with multi-signal flow graphs model, denotes the function models, fault patters and tests in a directional chart [6-7]. It has the advantages of both logic and information flow models. Besides the models above, researches present other models, such as graph pattern [8], structure model [9] and testability requirement model [10].

A proper model is needed when we analyze the measurability of ATE. Based on the model, the index parameters can be obtained to evaluate the measurability performance. After comprehensive consideration, we choose the information flow model which is simple and adequate to analyze the measurability of the ATE. The main reasons and more details are given in part $A$ of section 3 .

\section{B. Information flow model of testability}

The basic representation of the information flow model includes dependency and logical representation of the system being analyzed. An information flow model has two primitive elements: tests and fault-isolation conclusions. Tests include all the sources of information which can be used to determine the health of a system, such as abnormal phenomenon, data of BIT (built-in test), and response of the ATE input. In this paper, test is denoted by $t_{l}(l=1,2,3, \cdots)$. Each test is a Boolean variable with value of " 1 " or " 0 ". $t_{l}$ is " 0 " when its output is normal, and " 1 " on the contrary. $f_{k}$ represents the set of fault-isolation conclusions. "No fault" is treated as a special fault-isolation conclusion. If the system is fault-free, the value of $f_{k}$ is " 1 ", on the contrary is " 0 ". The input can be tested is denoted by Int.

Here is an example of information flow model shown in Fig.2 [14]. According to the model, the relations between test fault-isolation conclusions and tests can be described by the correlative matrix named FT matrix as shown in Table1.

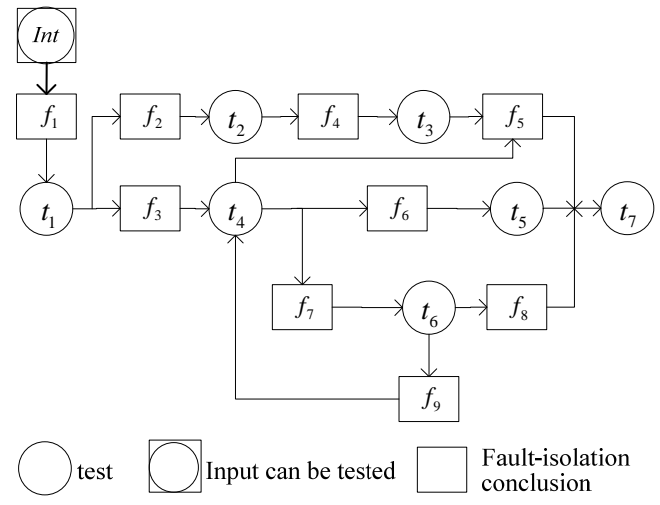

Fig.2. A system denoted by directional chart in information flow model

In the FT matrix, the rows refer to which test can observe the corresponding fault-isolation conclusion. When the conclusion existed, test represented by " 1 " in that row is abnormal.

The columns refer to which test and fault-isolation conclusion can be observed by the corresponding test. When one element of " 1 " corresponding fault-isolation conclusion has occurred, the column's test result is abnormal.

Table 1. The FT matrix of the information flow model

\begin{tabular}{ccccccccc}
\hline & Int & $t_{1}$ & $t_{2}$ & $t_{3}$ & $t_{4}$ & $t_{5}$ & $t_{6}$ & $t_{7}$ \\
\hline Int & 1 & 1 & 1 & 1 & 1 & 1 & 1 & 1 \\
\hline$f_{1}$ & 0 & 1 & 1 & 1 & 1 & 1 & 1 & 1 \\
\hline$f_{2}$ & 0 & 0 & 1 & 1 & 0 & 0 & 0 & 1 \\
\hline$f_{3}$ & 0 & 0 & 0 & 1 & 1 & 1 & 1 & 1 \\
\hline$f_{4}$ & 0 & 0 & 0 & 1 & 0 & 0 & 0 & 1 \\
\hline$f_{5}$ & 0 & 0 & 0 & 0 & 0 & 0 & 0 & 1 \\
\hline$f_{6}$ & 0 & 0 & 0 & 0 & 0 & 1 & 0 & 1 \\
\hline$f_{7}$ & 0 & 0 & 0 & 1 & 1 & 1 & 1 & 1 \\
\hline$f_{8}$ & 0 & 0 & 0 & 0 & 0 & 0 & 0 & 1 \\
\hline$f_{9}$ & 0 & 0 & 0 & 1 & 1 & 1 & 1 & 1 \\
\hline No Fault & 0 & 0 & 0 & 0 & 0 & 0 & 0 & 0 \\
\hline
\end{tabular}

\section{INFORMATION FLOW MODEL OF ATE MEASURABILITY}

A. Applicability of information flow in the measurability analysis of ATE

We found that the information flow model can be used to analyze the ATE measurability. The reasons are given as follows:

1) Relations between test and measure

Measure is a comprehensive concept includes test, calibration, metrology, verification and so on. For the ATE, the ability to be measured (or tested) can be verified by measure (here is calibration). So measure and test have some common grounds.

2) The logic relations of trace chain

The measurability of ATE means its ability to be calibrated (or measured). Calibration is a necessary process in which a standard instrument is used to insure the accuracy of ATE. There are two approaches to calibrate an ATE: One is carrying the ATE being calibrated to the reference institute or laboratory, and the other is on-station calibration 
which is done without moving any components of the ATE. Compare with the former approach, on-station calibration has many advantages as follows:

a. It takes less shutdown time;

b. The calibration process spends less time;

c. There's no need to remove and carry the components of the ATE being calibrated.

In order to take full advantage of the test resources in the ATE, the instrument with higher accuracy can be used to calibrate the instrument with lower accuracy. The unbroken trace chain is decided according to the precision of instruments. Fig. 3 is an example of trace chain. Here, the DMM (digital multimeter) is used to calibrate the digital oscilloscope by which the arbitrary waveforms generator is calibrated. The accuracy of these instruments is guaranteed in this way.

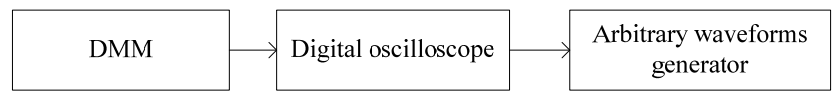

Fig.3. An example of trace chain

The trace chain is a mode of information flow. The quantity information is transferred from the higher to the lower instrument. The calibration between two instruments can be seen as a test. At the same time, the elements in the trace chain have logic relations. For example, if the digital oscilloscope in Fig. 3 is out of tolerance, it can't be used to calibrate the arbitrary waveforms generator. The logic information flow in the trace chain is the characteristic of correlative matrix.

3) The Boolean variable in the calibration

In the testability model, the conclusion of test has two states: normal or abnormal (fault or fault-free), which is denoted by " 0 ” and " 1 ” (Boolean variable) respectively. The calibration also has two states: in tolerance or out of tolerance. Here in tolerance can be seen as normal and out of tolerance represents abnormal.

\section{B. A practical example of information flow model for the ATE measurability}

In this representation, we define logic values for calibration and out-of tolerance conclusions [12]. The main idea of measurability information flow model is to determine the correlative matrix according to the conjunctive relations of trace chain.

There is a practical ATE which adopts the integrated technology of VXI and GPIB mixed BUS. Its hardware is shown in Fig.4. The ATE has many instruments: a DMM, a digital oscilloscope, a multifunctional module, an arbitrary waveforms generator, a timer, switch resources and accessories. These instruments have many ranges and modes. To ensure the accuracy of the ATE, all the instruments should be calibrated in a certain period of time.

First, we design the trace chain according to the accuracy of instruments for the ATE. As shown in Fig.5, the DMM is used to calibrate the digital oscilloscope and the other instruments are generally quite similar to this approach. According to the trace chain, we make good use of the internal test sources of the ATE and only need one more external calibration standard instrument in the highest rank of the trace chain.

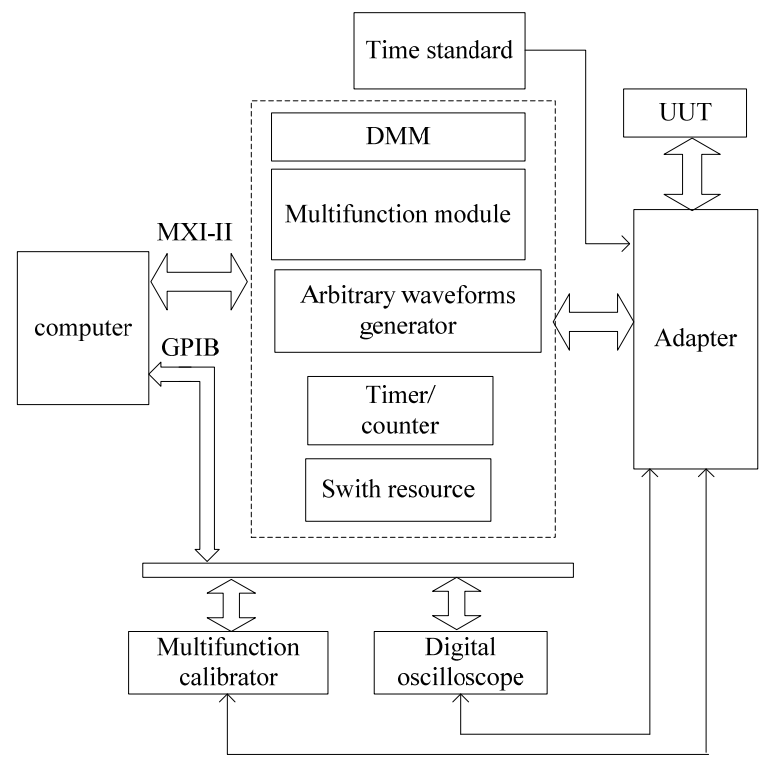

Fig.4. Hardware of the ATE

Two primitive elements in the information flow are the calibration and calibration out-of-tolerance conclusions. Let's consider that:

OT: The behavior of out of tolerance for an instrument being calibrated.

WT: The behavior of within-tolerance for an instrument being calibrated.

OC matrix: the correlative matrix of the OT conclusion and calibration.

$O C_{i j}$ : the element in the $i$-th row and $j$-th column of the $O C$ matrix.

$c_{j}$ : the calibration process between two adjacent instruments.

$o_{i}$ : the calibration conclusion of OT.

There are two conclusions of calibration: fail and pass. Pass means the instrument being calibrated is within tolerance. On the contrary, fail means the OT. The OC matrix is shown in Table2. Here " 1 " represents fail (OT) and " 0 " represents pass (WT). The detailed meaning of the $O C$ matrix is given as follows:

The rows of the $O C$ matrix refer to which calibration become abnormal when the corresponding instrument is out of tolerance. The abnormal conditions fall into two groups: one is that the calibration of the instrument is out of tolerance, and the other is lack of traceability. Take the OT of reference frequency standard $o_{7}$ for example. When $o_{7}$ is out of tolerance, the calibration $c_{6}$ which represents the calibration result between the external calibration standard instrument and the reference frequency standard is out of tolerance, and that is the first type of abnormality. At the same time, the calibration of $c_{7}$ is lack of traceability because of the poor accuracy for the reference frequency standard, and that is the other type of abnormal conditions. These logical relationships are: 


$$
o_{i} \Rightarrow \wedge_{j} C_{j} \quad\left(\text { if } O C_{i j}=1\right)
$$

where $\wedge$ is the logic AND.

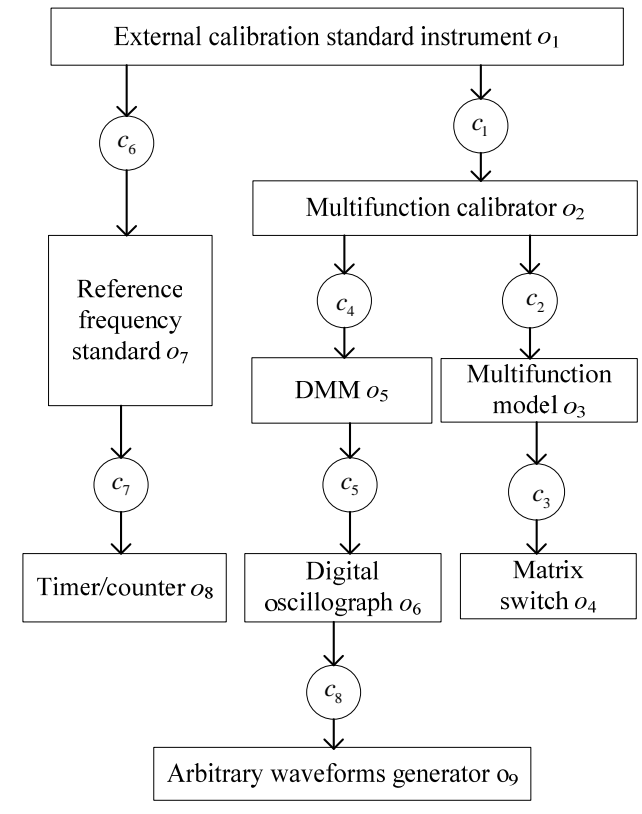

Fig.5. Information flow directional chart of the ATE trace chain

Table 2. Correlative matrix of calibration and OT conclusion

\begin{tabular}{ccccccccc}
\hline & $c_{1}$ & $C_{2}$ & $c_{3}$ & $c_{4}$ & $c_{5}$ & $c_{6}$ & $c_{7}$ & $c_{8}$ \\
\hline$o_{1}$ & 1 & 1 & 1 & 1 & 1 & 1 & 1 & 1 \\
$o_{2}$ & 1 & 1 & 1 & 1 & 1 & 0 & 0 & 1 \\
$o_{3}$ & 0 & 1 & 1 & 0 & 0 & 0 & 0 & 0 \\
$o_{4}$ & 0 & 0 & 1 & 0 & 0 & 0 & 0 & 0 \\
$o_{5}$ & 0 & 0 & 0 & 1 & 1 & 0 & 0 & 1 \\
$o_{6}$ & 0 & 0 & 0 & 0 & 1 & 0 & 0 & 1 \\
$o_{7}$ & 0 & 0 & 0 & 0 & 0 & 1 & 1 & 0 \\
$o_{8}$ & 0 & 0 & 0 & 0 & 0 & 0 & 1 & 0 \\
$o_{9}$ & 0 & 0 & 0 & 0 & 0 & 0 & 0 & 1 \\
\hline
\end{tabular}

The columns of $O C$ matrix explain that the corresponding calibration can verify which instrument is out of tolerance. When one element of " 1 " corresponding calibration result of OT occurred, the column's calibration will be abnormal. These logical relations can be shown as:

$$
\neg C_{j} \Rightarrow\left(\hat{i}_{i} \neg O_{i}\right) \quad\left(\text { ifOC } C_{i j}=1\right)
$$

where $\neg$ is the logic NOT.

\section{RESULTS ANALYSIS OF MEASURABILITY MODEL}

Having established the $O C$ matrix, we can calculate some typical measurability indexes of the ATE given as follows:

Undetected OT set: the OT which can not be observed by any of the available calibrations. In fact, the undetected OT is lack of traceability and is defined as:

$$
U O=\left\{o_{i} \mid O_{i}=0, \forall i\right\}
$$

where $O_{i}$ means the vector of the $i$-th row. We can see from Table2 that there is not any of undetected OT sets.

Contribution Rate of Measurability (CRM): the weighting of the measurability indexes. For the OT conclusion of $o_{i}$, the CRM can be obtained by:

$$
\frac{\sum_{j} O C_{i j}}{\sum_{i j} O C_{i j}} \times 100 \%
$$

The CRMs of all the instruments in the ATE are shown in Table3. The higher the CRM, the more attention should be given when we analyze the measurability of the ATE. We can see that the CRM of external calibration standard instrument is the highest which comes out to $30.8 \%$. It means that this instrument is the most important effect factor for the ATE measurability. Ensuring the accuracy of the external calibration standard instrument is the premise of ATE calibration. In addition, the CRM of the multifunction calibrator is high because more instruments are traced to it. The CRM can be used to analyze the key instrument, determine calibration interval and study the stability of ATE.

Table 3. Contribution rates of measurability

\begin{tabular}{cc}
\hline Instruments & CRM [\%] \\
\hline Superior standard instrument & 30.8 \\
Multifunction calibrator & 23.1 \\
Multifunction module & 7.7 \\
Matrix switch & 3.8 \\
DMM & 11.5 \\
Digital oscilloscope & 7.7 \\
Reference frequency standard & 7.7 \\
Timer & 3.8 \\
Arbitrary waveforms generator & 3.8 \\
\hline
\end{tabular}

Calibration Interval: There are two traditional methods to determine the calibration interval of ATE: one is taking the highest instrument of the trace chain as the standard of the ATE calibration interval; the other is to take the shortest calibration interval of all the instruments. Both of them have some disadvantages: the former approach is one-sided and the latter wastes time and resources. We suggest a more reasonable method. Firstly, we determine the calibration interval of each instrument in the ATE, and then take the CRM as the weighting to get the weighted average of calibration interval. The final result is the uniform calibration interval of ATE system. In order to guarantee the accuracy, we can use the internal instrument to calibrate others between the periods of calibrations.

Redundant Calibration: a form of excess calibration. A calibration is considered excess when some combination of other calibrations provides the same information it does. Compare each column of the $O C$ matrix, if $C_{i}=C_{j}(i \neq j)$, 
the corresponding calibrations are redundant calibrations of each other. There isn't any redundant calibration in Table 2.

Hidden OT Set ( $\mathrm{OH}$ set): the case where the root cause is masked by the observed symptoms. An instrument which is out of tolerance has the same characteristic with multiple instruments. The existence of hidden OT set causes the omission of instrument's OT. There is a way to find the hidden set: for the OT conclusion $o_{k}$, the hidden OT set is the remaining after removing the rows whose element of column is " 1 ". That is

$$
O H=\left\{v_{i} \mid O C_{i j}=0, \text { if } O C_{j k}=0\right\}
$$

Any of the OT conclusions in the $\mathrm{OH}$ and $o_{k}$ occurring at the same time will bring about the hidden OT.

Taking $o_{7}$ for example, when the reference frequent standard is out of tolerance, the accuracy of the timer's value can't be guaranteed. That means the hidden OT set of $o_{7}$ is $\left\{o_{8}\right\}$. The hidden OT set of $o_{k}$ is shown in Table 4 .

Table 4. Hidden OT sets of OT conclusions

\begin{tabular}{cc}
\hline OT Conclusion & Hidden OT Set \\
\hline$o_{1}$ & $O_{2}, O_{3}, O_{4}, O_{5}, O_{6}, O_{7}, O_{8,}, O_{9}$ \\
$o_{2}$ & $O_{3}, O_{4}, O_{5}, O_{6}, O_{9}$ \\
$O_{3}$ & $o_{4}$ \\
$o_{5}$ & $o_{6}, O_{9}$ \\
$o_{7}$ & $o_{8}$ \\
$o_{6}$ & $o_{9}$ \\
\hline
\end{tabular}

Measure Coverage Rate: If all the calibrations are done, the coverage rate is $100 \%$. For instance, when $c_{7}$ is absent, the coverage rate is

$$
\frac{\text { number of calibrations being done }}{\text { total number of calibrations }}=\frac{7}{8}=87.5 \%
$$

\section{CONCLUSION}

In this paper, the concept of measurability was first investigated and its key indexes are introduced. The flow information model is used to analyze the measurability of the ATE. An example is used to illustrate the efficiency and maneuverability. The approach may be useful in the design, analysis and evaluation of the ATE measurability.

This paper focuses on the evaluation process of the measurability. In our opinion, measurability is a design concept, and there should be some guides when we design a measure or test equipment. This is another topic we want to analyze and solve in the next step.

Furthermore, the concept of test is consists of ETE (external test equipment, most are ATE) and BIT (built-in test). This paper only considered the measurability of the ATE. In the future, we will also study the measurability of BIT and extend the measurability for engineering applications.

\section{REFERENCES}

[1] Shakei, M. (1996). Advances in system fault and diagnosis. University of Connecticut.

[2] Sheppard, J.W., Simpson, W.R. (2007). A mathematical model for integrated diagnostics. Design \& Test of Computer, 8 (4), 25-38.

[3] Azam, M., Tu, F., Pattipati, K.R., Karanam, R. (2004). A dependency model-based approach for identifying and evaluating power quality problems. IEEE Transactions on Power Delivery, 19 (3), 1154-1166.

[4] Sheppard, J.W., Kaufman, M.A. (2005). A bayesian approach to diagnosis and prognosis using built-in test instrumentation and measurement. IEEE Transactions on Computer, 54 (3), 1003-1018.

[5] Luo, J.H., Ghoshal, S., Pattipati, K.R. (2008). Distributed fault diagnosis using dependency modeling without revealing sub-system details. In Aerospace Conference, 1-8 March 2008. IEEE, 1-10.

[6] Gould, E. (2004). Modeling it both ways: Hybrid diagnosis modeling and its application to hierarchical system designs. In AUTOTESTCON 2004, 20-23 September 2004. IEEE, 576-582.

[7] Lv, X.M., Huang, K.L., Lian, G., Xue, K. (2008). Design and analysis of comples system testability based on hybrid diagnostic model. Journal of Projectiles, Rockets, Missiles and Guidance, 28 (6), 283-287. (in Chinese)

[8] Liu, B.D., Hu, C.H. (2006). Testability analysis of analog circuit fault based on graph pattern. Journal of Projectiles Guidance, 27 (4), 257-260. (in Chinese)

[9] Lian, G.Y., Huang, K.L., Guo, R., Jiang, Y.H. (2007). Research on testability design and analysis technology based on structure model. Systems and Electronics, 29 (10), 1777-1780.

[10] Su, Y.D., Liu, G.J., Qiu, J. (2010). DSPN-based testability requirement modeling and analysis of phased-mission systems. Systems Engineering Theory \& Practice, 30 (7), 1272-1278.

[11] Yadav, S., Gupta, V.K., Bandyopadhyay, A.K. (2010). Investigations on measurement uncertainty and stability of pressure dial gauges and transducers. Measurement Science Review, 10 (4), 130-135.

[12] Sheppard, J.W., Simpson, W.R. (1992). Applying testability analysis for integrated diagnostics. IEEE Design \& Test of Computers, 9 (3), 65-78.

[13] Yang, P., Qiu, J., Liu, G.J. (2008). Research on extended dependency model-based testability analysis. Systems Engineering and Electronics, 30 (2), 371-374.

[14] Wen, X.S., Xu, Y.Ch., Yi, X.Sh., Chen, X. (2002). Theory and Application of Intelligent Built-in Test. Beijing: National Defense Industry Press, 57-63.

[15] Song, J., Vorburger, T., Thompson, R. et al. (2010). Three steps towards metrological traceability for ballistics signature measurements. Measurement Science Review, 10 (1), 19-21

Received December 12, 2011. Accepted August 3, 2012. 